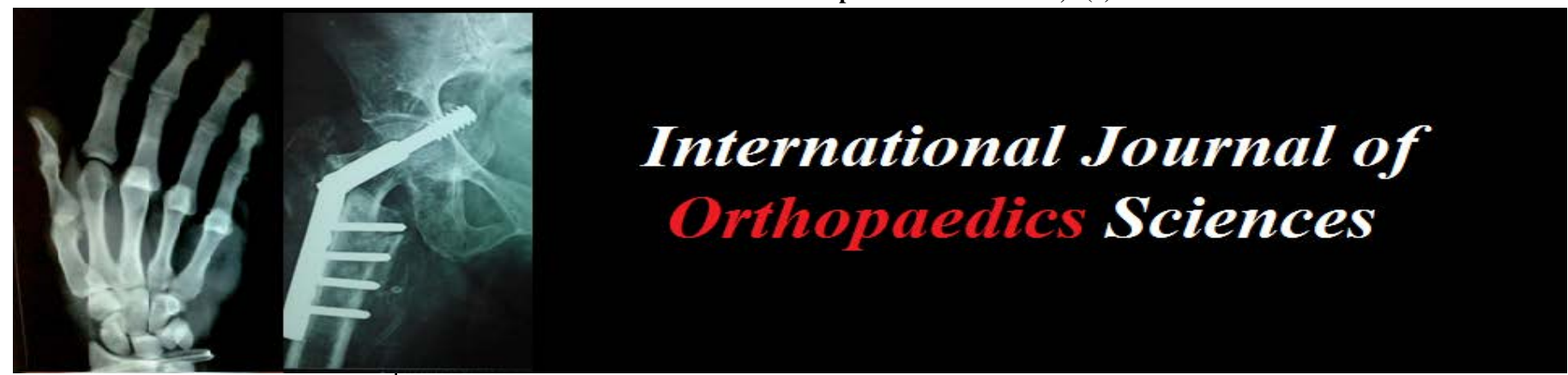

E-ISSN: 2395-1958

P-ISSN: 2706-6630

IJOS 2021; 7(3): 298-302

(C) 2021 IJOS

www.orthopaper.com

Received: 01-05-2021

Accepted: 03-06-2021

Dr. Kurmana Vamsi Krishna Assistant Professor, Department of Orthopaedics, Gems Hospital, Ragolu, Andhra Pradesh, India

Dr. Tutika Dinesh Kumar

Assistant Professor, Department of Orthopaedics, Gems Hospital, Ragolu, Andhra Pradesh, India

Dr. Deepak Chamalla

Assistant Professor, Department of Orthopaedics, Gems Hospital, Ragolu, Andhra Pradesh, India

Corresponding Author: Dr. Kurmana Vamsi Krishna Assistant Professor, Department of Orthopaedics, Gems Hospital, Ragolu, Andhra Pradesh, India

\section{Evaluation of functional outcome of intracapsular fracture neck of femur with Austin Moore prosthesis at a tertiary care hospital in Srikakulam}

\author{
Dr. Kurmana Vamsi Krishna, Dr. Tutika Dinesh Kumar and Dr. Deepak \\ Chamalla
}

DOI: https://doi.org/10.22271/ortho.2021.v7.i3e.2760

\begin{abstract}
Introduction: Fractures of the femoral neck are common injuries occurring in the elderly population due to osteoporosis. They present a significant challenge to Orthopaedic surgeons because of the high rate of complications like nonunion, avascular necrosis, and associated comorbidities. Historically, Austin Moores prosthesis has served as an exemplary implant over the years in the management of intracapsular fracture neck of femur in older individuals. Our aim of the study is to asses postoperative function in these population and to validate the use of AMP in current Orthopaedic practice.

Methods \& Materials: This is a hospital-based cohort study. We did this study in thirty patients who had the fractured neck of the femur using Austin Moore Prosthesis, who are admitted to the Department of Orthopaedics in Great Eastern Medical School \& Hospital, Srikakulam from Dec 2017 to Dec 2019. All patients were followed for twelve months. It is a patient-reported outcome study based on a questionnaire using a Harris hip score.

Results: Harris hip score, which is the gold standard in the assessment of postoperative function in hip surgeries was used in this study. We graded the patients as excellent, good, fair, and poor depending on the functional outcome based on each criterion in this scoring system. The functional results were excellent in $50 \%$, good in $23.3 \%$, fair in $23.3 \%$, and poor in $3.3 \%$ of cases. The poor results are due to moderate hip pain after surgery. The complication rate was low.

Conclusion: Hemiarthroplasty with Austin Moore Prosthesis proved to be a good choice for the management of fracture neck femur in the elderly population with limited physical demand and low economic background. The mortality and morbidity are low; the operative procedure is simple with satisfactory functional results and fewer complications.
\end{abstract}

Keywords: fracture neck of femur, Austin Moore prosthesis

\section{Introduction}

Fractures around the hip are common and comprise around $20 \%$ of operative cases in Orthopaedic unit ${ }^{[1]}$. Of those cases, intracapsular fractures of the femoral neck account for about $50 \%$ of cases. According to doruku et al, a recent surge in the incidence of hip fractures can be attributed to increased life expectancy worldwide, and it is estimated that there will be an increase in cases from 1.6 million in 1990 to about 6.2 million in $2050^{\text {[2] }}$.

The problem of fracture neck of femur is one of the oldest in orthopedics. Inspite of numerous advances in osteosynthesis, the incidence of nonunion and avascular necrosis is very high. There is higher rate of non-union (5\%) and osteonecrosis (10\%) associated with fracture neck of femur in un-displaced fractures. In displaced fractures following internal fixation, the nonunion rate is $10-30$ per cent and osteonecrosis is 15 -33 percent, Fractures of the femoral neck still remain an unsolved fracture to the orthopedic surgeon as far as treatment and results are concerned. Fractures of the femoral neck can occur at all ages and in both sexes. The most common mechanism of injury is simple fall with force along the femoral neck through greater trochanter, causing fracture ${ }^{[3]}$. The incidence of these cases is increased mainly due to an increase in trivial trauma, osteoporosis and other comorbidities in the elderly population.

Management techniques for femoral neck fractures in elderly patients have been controversial due to the risk of complications like nonunion and osteonecrosis of the femoral head. 
Posture - related complications like bedsores, deep vein thrombosis resulting in secondary pulmonary embolism are associated with high mortality and morbidity. when compared to internal fixation, The advantages of uncemented Austin Moore Self-Locking Prosthesis (AMP) include less operative time, less blood loss and fewer postoperative complications. It is also cost-effective. when compared to cemented hemiarthroplasty, uncement AMP hemiarthroplasty $\mathrm{Y}$ has better bone to implant union, no risk of bone cement implantation syndrome, easy removal in case of infection. Austin Moore Hemiarthroplasty is commonly done in developing countries like India. It is most commonly reserved for non-ambulatory people ${ }^{[4]}$

Daniel et al. ${ }^{[6]}$ in their study concluded that the patients treated with Austin Moore prosthesis returned to preinjury level of activity with satisfactory outcome Manzoor et al. ${ }^{\text {[7] }}$ in their study concluded that prosthetic replacement with AMP is an appropriate mode of treatment in elderly patients in whom early mobilization is essential to prevent complications of prolonged immobilization.

This study evaluates functional outcome of hemiarthroplasty with AMP in elderly patients at our tertiary care center.

\section{Patients and Methods}

Our study has been carried out in 30 patients with a fracture neck of the femur using Austin Moore prosthesis, admitted in the department of Orthopaedics in great eastern medical school \& hospital, Ragolu, Srikakulam from December 2017 to December 2019. All patients were followed up for one year.

\section{Inclusion Criteria}

- Patients aged 60 and above.

- Nonunion femoral neck fracture without hip joint arthritis $<3$ months old

- Pauwells type 2, 3

- Garden 3,4

\section{Exclusion Criteria}

- Patients below age of the 60 years

- Pathological fractures, open fractures and fractures with neurovascular injuries

- Patients who were unfit for procedure due to medical comorbidities.

- $\quad$ Pauwels type 1 , garden type 1,2

- Neglected Nonunion femur neck fracture with hip joint artritis grade 4

We took permission from the institutional ethical committee in our hospital, and well-written and informed consent was taken from all the patients and their relatives who were participating in the study. We did a thorough clinical examination and detailed history about their pre-fracture ambulatory status and other medical comorbidities. Skin traction was applied in all fresh fractures preoperatively to reduce pain and muscle spasm. Proper preoperative anaesthetic assessment was done after lab investigations and radiographs.

\section{Surgical Procedure}

All the surgeries were performed on an elective basis within first 5 days of trauma with aseptic precautions under spinal or epidural anesthesia. All surgeries were done with patient in lateral decubitus position. Modified posterolateral approach was used in all cases. short external rotators divided t shaped capsulotomy done and head was extracted and head size was measured using gauge. lateral entry point made and serial broaching was done and canal was irrigated with normal saline and gentamicin. Hemiarthroplasty was done using Austin Moore prosthesis of appropriate size and took proper precautions like lateral entry and centralized stem and bone plugs in amp stem to avoid surgical complications. Hip was tested for a full range of movements and stability was checked intraoperatively using chuck test and telescoping. We repaired short external rotators using the anchoring technique.

Postoperatively all patients were allowed weight bearing as tolerated, usually 24 to 72 hours after surgery, depending on the patient compliance using a walker. Antibiotics were administered postoperatively for three days to prevent infection. We discharged all the patients after a minimum of ten days.

We advised the patients to avoid squatting and to use the western toilet.

Regular follow up of the patients was done at two weeks, six weeks, three months, six months and 12 months. At each follow-up, the patients were assessed clinically using the Harris Hip Score and radiologically to detect any loosening, heterotrophic ossification, subsidence of the Prosthesis and protrusioacetabuli.

In this study harris hip scoring system used where total of 100 points were used with 70 poor 70 -79 fair 80-89 good 90-100 excellent

Statisyical anlaysis: Our Studydesign is prospective observational study and Sample size is 30 Analysis was done by using MS excel. Qualitative data represented as percentages and quantitative data was represented as means and standard deviation. Statistical analysis was done using chi square test and significance was assessed by p value

\section{Results}

The mean age of patients was about 69.43 years, ranging from $60-89$ years, and about $50 \%$ of the patients belonged to the 60-69 age group.

In our study, 17 patients were female, and 13 were male. The laterality of the fracture was slightly more with the left side, with 53\% (16patients). The injury mode was mainly with trivial fall, i.e., fall during walking or slipping in the bathroom, which constitutes about $90 \%$ and only $10 \%$ were due to road traffic accidents. $39-55 \mathrm{~mm}$ sizes of Prosthesis were used as per requirement and $40 \%$ of cases have been done with 47 size implant, followed by 45 sizes with $30 \% .43$ size $20 \%$ 39, 51, 55 size $3.3 \%$ each

Harris Hip scores of our 30 operated patients averaged 86 (range, 69-98) at final follow-up after 12 months. We achieved excellent results in 50\% (15 patients), good in $23.3 \%$ (7 patients), and fair in $23.3 \%$ (7 patients). Only $3.3 \%$ (1 patient) had poor results (coexistent osteoporosis) In our study group, one patient developed a superficial infection, which was managed conservatively, another patient suffered deep vein thrombosis. One patient had superficial bed sore managed with airbed and daily dressing. No loosening, osteolysis, dislocations, periprosthetic fractures, deep infection, and residual anterior thigh pain were reported. $60 \%$ were able to ambulate after three days, and $20 \%$ took one week, remaining $20 \%$ took two weeks. 
Table 1: Showing complications

\begin{tabular}{|c|c|c|}
\hline Complications & No of Patients & Percentage \\
\hline None & 27 & 90 \\
\hline Superficial Infection & 1 & 3.3 \\
\hline Bed Sores & 1 & 3.3 \\
\hline DVT & 1 & 3.3 \\
\hline
\end{tabular}

Table 2: Showing Final Harris Hip Score and clinical result

\begin{tabular}{|c|c|c|c|}
\hline Grade & Harris Hip Score & No. of Patients & \% \\
\hline Excellent & $90-100$ & 15 & 50 \\
\hline Good & $80-89$ & 7 & 23.3 \\
\hline Fair & $70-79$ & 7 & 23.3 \\
\hline Poor & $<70$ & 1 & 3.3 \\
\hline
\end{tabular}

The final harris hip score was excellent in 50 percent of patients and good in 7 patients fair in 7 patients poor in 1 patient with a chi square value of 13.2 and it is statistically significant with a $\mathrm{P}$ value of $0.0042(<0.05)$

\section{Discussion}

Femoral neck fractures is still a controversial topic regarding the treatment as results have been variable with various treatment modalities, including internal fixation, Hemi replacement, and total hip replacement.

Our study's mean age is 69.43, and the average age was 66 years in Saxena and Saraf group ${ }^{[8]}, 67$ years in Anil B Dhule ${ }^{[9]}$ and 69 years in Shan SA group ${ }^{[10]}$ and 65 years by Essoh et al., ${ }^{[11]}$ mean age in our study is similar to other studies in the literature

In our study, the incidence was more in the female group, with $57 \%$ compared to $83 \%$ in Essoh J.B group ${ }^{[11]}$, 62.5\% in the Freeman ${ }^{[12]}$ group, $57 \%$ in study by Syed SN ${ }^{[13]}$. The leftsided hip involvement was about 53\%, and it is similar when compared to Boyd and Salvatore group ${ }^{[14]}$. A similar finding was noted by Kishore Roy ${ }^{[15]}$ with $68 \%$. About $90 \%$ of our study population have been involved in an injury due to fall, and it is identical to Gyepes ${ }^{[16]}$ and Ingalhalikar ${ }^{[17]}$.

Among all study subjects, $50 \%$ of the patients had at least one or more systemic comorbidity, the most common being hypertension, seen in six patients. In contrast, other studies reported 33\%, and 28.6\% patients with hypertension by Karen Amit ${ }^{[18]}$ and Mue Daniel ${ }^{[6]}$ respectively.

The mean average duration of hospital stay was two weeks, similar to the study by Mue Daniel [6] (2015) with a mean duration of 16 days. In this study, $10 \%$ of patients developed minor complications. There were no significant complications. Posterior dislocation is reported in many series. Salvatti et al. ${ }^{19}$ in their study explained that limb kept in flexion and adduction is the most common cause for dislocation of Prosthesis and reported 2.8\% posterior hip dislocations in their study. Karen Amit ${ }^{[18]}$ in 2014 reported $1.25 \%$ had a dislocation in his studies. No postoperative dislocations were reported in our study

They have been compared with other groups, and our results were satisfactory and are within the range of other studies. We had about 50\% excellent results and 3.3\% with poor outcomes.

In 2002, Parker ${ }^{[20]}$ et al. in a review of 243 Austin Moore prostheses found at 1year post-surgery, 61 patients (25.1\%) had residual pain, and 17 patients $(7 \%)$ required revision surgery for aseptic loosening. Both residual pain and revision for aseptic loosening were strongly associated with features of the operative technique, based on the resection level of the femoral neck, seating of the prosthesis, prosthetic head size. They stated that most important predictor of a poor outcome was the failure to seat the collar of the prosthesis on the calcar.

In 2004, W.P.Yau et al. ${ }^{\text {[21] }}$ concluded in their study that AMP is a useful operation in the management of intracapsular fracture neck of femur in geriatric age group and alternate methods should be chosen for relatively younger patients. (internal fixation, total hip replacement)

In 2006, Alan R.Norrish et al. ${ }^{[22]}$ reported acceptable longterm results for an uncemented Austin Moore Hemiarthroplasty in their study. They stated that prosthesis is inexpensive and does not suffer from the disadvantages of using bone cement for implantation in elderly people. In 2006, Masson et al., ${ }^{[23]}$ in their prospective randomized comparative study compared the various treatment modalities in displaced intracapsular fracture neck of femur in the elderly. They concluded that the osteosynthesis produced a high rate of revision surgery and an inferior functional outcome compared with that of Hemiarthroplasty.

In 2010, Abraham O. Odumala et al. ${ }^{[24]}$ stated in their study that closed reduction after dislocation of the unipolar prosthesis has a higher failure rate significantly in dementia patients. They noted that the girdle stone procedure was considered after the first dislocation in these patients.

In 2010, Nader N.T. Rehmatullah et al. ${ }^{\text {[25] }}$ showed an inverse correlation between prosthesis head size and metaphyseal fit in their study. They stated that AMP with head sizes greater than $49 \mathrm{~mm}$ are likely to be loose, and in such cases, the use of an uncemented bipolar or cemented Hemiarthroplasty is recommended.

In 2012, M.Hossain et al. ${ }^{[26]}$ stated in their study that the risk of perioperative death was significantly higher following cemented implant insertion. They reported that mortality risk was exacerbated in patients with pre-existing cardiovascular morbidity.

In 2014, Anil B.Dhule et al. ${ }^{[9]}$ concluded in their study that minimal incision surgery reduces the duration of surgery, blood loss, and postoperative pain. They used a modification of the posterolateral approach to preserve short external rotators that provide increased hip joint stability and better results. Karen Amit et al. ${ }^{[18]}$ concluded that Hemiarthroplasty with uncemented AMP is a safe procedure with a low incidence of complications but should be reserved for elderly patients.

In 2015, daniel $\mathrm{m}$ et al. ${ }^{[6]}$ in their study, concluded that Austin Moore Hemiarthroplasty in elderly patients gave satisfactory results with minimal morbidity. They stated that careful patient selection is essential and may decrease complications rate and improve the results.

In 2017, Matthew $\mathrm{J}$ et al. ${ }^{[27]}$ concluded that there is no advantage of the bipolar prosthesis over unipolar in reoperation rate. In 2017, G.Mamarelis et al. ${ }^{[28]}$ reported that return to theatre within 30 days of Hemiarthroplasty for femoral neck fractures is associated with a longer hospital stay, higher re-admission rate, and higher revision rates. It may be a useful short term quality indicator for long term outcome measure.

In 2018, Goyal et al. ${ }^{[5]}$ declared in their study that Austin Moore Hemiarthroplasty showed better functional results. He stated that patients need to modify their daily routine activities and preferably avoid squatting and sitting crosslegged on the floor for more than ten years of AMP prosthetic life.

In 2018, balaji et al. [37] concluded that compared to uncemented Austin Moore prosthesis, cemented bipolar prosthesis has more total blood loss and duration of surgery. 
HA is still a very good option for treating fracture neck of femur in elderly patients especially in developing countries like India. AMP is the choice of implant in less active patients.

In 2019, Pulkit Jain et al. ${ }^{\text {[29] }}$ concluded in their study that though unipolar AMP faces criticism for acetabular wear, most patients showed excellent to good results and is a good choice in geriatric patients.

In 2020, Cui et al. ${ }^{[30]}$ in their meta-analysis of 7 randomized controlled trials and five cohort studies, concluded that in the treatment of elderly femoral neck fractures, the internal fixation group has lesser operative time and less bleeding, and the perioperative advantage is more pronounced. However, the Hemi-replacement group had more advantages in postoperative functional scoring and reoperation.

In 2020, Farey et al. [31] concluded that unipolar Hemiarthroplasty works better for patients with shorter life expectancy than bipolar Hemiarthroplasty.

Table 3: Showing Functional Results in Various Studies

\begin{tabular}{|c|c|c|c|c|c|}
\hline Investigator & No. of Patients & Excellent & Good & Fair & Poor \\
\hline SALVATI et al (1964) ${ }^{[19]}$ & 251 & 31 & 26 & 25 & 8 \\
\hline SAXENA AND SARAF (1978) ${ }^{[8]}$ & 82 & 46.1 & 44.8 & 6.5 & 2.6 \\
\hline R Kumar01 (1980) ${ }^{[36]}$ & 25 & 28 & 36 & 20 & 16 \\
\hline Bavadekar and Manelkar (1987) ${ }^{[35]}$ & 328 & 60 & 30 & 10 & 0 \\
\hline BG Dubani (2004) ${ }^{[33]}$ & 123 & 38.2 & 34.1 & 17.8 & 9 \\
\hline PS Maini (2006) ${ }^{[32]}$ & 271 & 54.2 & 21 & 10.7 & 3.7 \\
\hline Laghari et al. (2014) ${ }^{[34]}$ & 50 & 44 & 27 & 20 & 9 \\
\hline Daniel et al.(2015) ${ }^{[6]}$ & 35 & 23 & 46 & 25 & 6 \\
\hline Pulkit Jain (2019) ${ }^{[29]}$ & 95 & 12 & 61 & 19 & 3 \\
\hline Present Study & 30 & 50 & 23.3 & 23.3 & 3.3 \\
\hline
\end{tabular}

\section{Limitations of the study}

1. Single centre, non-randomized, non-controlled, prospective, study

2. Small sample size with short duration of follow up

\section{Conclusion}

We conclude that Hemiarthroplasty with Austin moores prosthesis for the fracture neck of femur is a good option in elderly patients, especially in the economically backward group, as it is cost-effective. The complications are less disabling. Weight-bearing is early and The final harris hip score was excellent in 50 percent of patients and good in 7 patients fair in 7 patients poor in 1 patient with a chi square value of 13.2 and it is statistically significant with a $P$ value of $0.0042(<0.05)$

\section{References}

1. Singer BR, McLauchlan GJ, Robinson CM, et al. Epidemiology of fractures in 15,000 adults: The influence of age and gender. J Bone Joint Surg $\mathrm{Br}$. 1998;80(2):243-248.

2. Doruk H, Mas MR, Yildiz C, et al. The effect of the timing of hip surgery on the activity of daily living and mortality in the elderly. Arch Gerontol Geriatr. 2004;39(2):179-185.

3. Linten P. Type of displacement in fractures of femoral neck and observations on fractures' impaction. J Bone Joint Surg 1949;34B:184-189.

4. Swiontowski MF. Intracapsular fractures of the hip. J Bone Joint Surg Am 1994;76-A:129-138.

5. Nandkishor B Goyal, Swapnil A Patil, Ajay A Nayakawadi: Fracture neck of femur treated with Austin Moore prosthesis - 10 year follow up MedPulse Research \& Publication 2018;8(3)

6. Mue Daniel, Salihu Mohammed, Awonusi Francis, Yongu William, Kortor Joseph, Elachi Cornilius. Early results of Hemiarthroplasty in elderly patients with fracture neck of femur.Nigerian medical journal 2015;56(1).

7. Basharat Manzoor, Shehzad Mehmood, Javed Iqbal M. Femoral neck fracture treated with Hemiarthroplasty by using Austin Moore Prosthesis-surgical outcome
Professional Med J 2018;25(12):1805-1808.

8. Saxena PS, Saraf JK. Moore Prosthesis in fracture neck of femur. Indian Journal of Orthopaedics 1978;12:138145.

9. Dhule AB, Ansari A, Patil A. Minimal Incision Surgery for Austin Moore Prosthesis in Intracapsular Fracture Neck Femur. Journal of Trauma \& Orthopaedics 2015;10(1):18-20

10. Shah SA, Memon A, Pirwani M. Outcome of Femoral Neck Fracture Treated by Austin - Moore Hemiarthroplasty in Elderly Patients. Med Forum 2015;26(1):45-49.

11. Essoh JB, Sie MD, Aka D et al. Austin Moore Hemiarthroplasty for Displaced Neck fracture in Patients Aged 55 Years and above. An Ivorian Experience. NJOT, 2006;1(8):13-12.

12. Freeman MAR, Todd RC, Pirie EJ. The role of fatigue in the pathogenesis of senile femoral neck fractures. JBJS 1974;56B:698-702.

13. Syed SN, Niaz H, Imran J. Outcome of Austin Moore Hemiarthroplasty in Elderly patients with femoral neck fractures. The journal of Pakisthan orthopedic Association 2010;22:14-19.

14. Boyd HB, Salvatore JE. Acute fractures of the femoral neck: Internal fixation or Prosthesis? JBJS 1964;46A:1066-1068.

15. Kishore Roy. Management of Intra Capsular Fracture Neck of Femur with Austin Moore's Hemiarthroplasty” A Clinical Study. Indian Journal of Applied Research. 2014;4(12):371-75.

16. Micheal Gyepes, Harry Z, Mellins, Isadore Katz. The low incidence of fracture of the hip in the Negro. JAMA 1962;181(12):1073-1074.

17. Ingalhalikar VT, Shekar Kumta. Fracture neck femur anatomical and biomechanical aspects. Clinc.Orthop. India The Journal of Pakistan orthopaedisc association 1987;20:14-19

18. Amit K, Yaron B, Alexander L, Rostislav N, David R. Functional Outcome after Hemi-Replacement for Femoral Neck Fracture (Subcapital Fracture) with Austin Moore Prosthesis. J Trauma Treat 2014;3:188.

19. Salvatti EA, Artz T, Algeitti P, Asins SE. Endoprosthesis 
in the treatment of femoral neck fractures. Orthop. clinc. North America 1974;5:757-777.

20. Khalid M, Sharif, Martyn J Parker. Austin moore Hemiarthroplasty -technical aspects and their effects on outcome, in patients with fracture neck of femur Injury, Int J. Care Injured 2002;33:419-422.

21. Yau WP, Chiu KY. clinical radiological analysis after Austin Moore hemiarthroplasty 2004, 35(10)

22. Alan R Norrish, Janardhan Rao, Martyn J, Parker. prosthesis survivorship and clinical outcome of the Austin Moore Hemiarthroplasty: An 8 yr mean follow up of a consecutive series of 500 patients. Injury 2006, 37(8).

23. Keating JF, Grant A, Masson M, Scott NW, Forbes JF. Randomized comparison of reduction and fixation, bipolar Hemiarthroplasty, and total hip arthroplasty in the treatment of displaced intracapsular hip fractures in healthy older patients. J Bone Joint Surg Am 2006;88:249-60. [PubMed] [Google Scholar

24. Abraham O, Odumala, Mohammed R Iqbal, Robert G. Middleton: Failure of closed reduction after dislocation of AMP: An analysis of risk factors: $6 \mathrm{yr}$ follow up study.

25. Nader Neil Terry Rehmatullah, Andrew MacDonald, Christopher Ingham, Benjamin. J. Davis : The relationship of head size vs metaphyseal fit with the Austin Moore Hemiarthroplasty; European Orthopaedics and Traumatology 2010;1(1):17-19.

26. Hossain M, Andrew JG. Is there a difference in perioperative mortality between cemented and uncemented implants in hip fracture surgery? ; Injury 2012;43(12):2161-2164

27. Matthew J Grosso, Jonathan R Danoff, Taylor S Murtaugh, David P Trofa, Andrew N Sawires, William B Macaulay. Hemiarthroplasty for Displaced Femoral Neck Fractures in the Elderly Has a Low Conversion Rate,The Journal of Arthroplasty 2017;32(1):150-154. ISSN 08835403

28. Mamarelis G, Key S, Snook J, Aldam C. Outcomes after early return to theatre following Hemiarthroplasty for intracapsular fractures of femoral neck ; The bone and joint journal 2017;99-B(7)

29. Pulkit Jain, Najmul Huda, Ajay Pant, Pratyush Parag. Austin moore prosthesis: Still the flag ship ? JBJD 2019;34(1).

30. Cui S, Wang D, Wang X et al. The choice of screw internal fixation and Hemiarthroplasty in the treatment of femoral neck fractures in the elderly: a meta-analysis. J Orthop Surg Res 2020;15:433

31. Farey, John E. MBBS(Hons), MMed(ClinEpi)1,a; Cuthbert, Alana R. BMathSc(Hons)2; Adie, Sam MBBS, MPH, PhD, FRACS(Orth)3; Harris, Ian A. MBBS, MMed(ClinEpi), PhD, FRACS(Orth)1,2,4 Revision Risk After Unipolar or Bipolar Hemiarthroplasty for Femoral Neck Fractures, The Journal of Bone and Joint Surgery: 2020 - Volume Latest Articles - Issue 10.2106/JBJS.20.00486

32. Maini PS, Navin Talwar, NIjhawan VK, Manish Dhawan. Results of cemented bipolar Hemiarthroplasty for fracture of the femoral neck-10 years study; IJO, July 2006;40(3):154-156.

33. Dudani BG, Azam SM, Madukeshwar GV. Bipolar Hemiarthroplasty for fracture of the neck of femur in the elderly; IJO, January 2004;38(1):12-15.

34. Laghari MA, Pahore MK, Maher IK, Arain MS. Femoral neck fracture; the outcome of unipolar Hemiarthroplasty in elderly patients. Professional Med J. 2014;21(5):874878.

35. Bavadekar AV. A Review of internal fixation and prosthetic replacement for fresh fractures of the femoral neck. Clini Orthop India 1987, 43-52.

36. Kumar R, Singh T. Early Results of Prosthetic Replacement in Old Neglected Cases of Fracture Neck femur. Indian Journal of Orthopaedics 1980;14:38

37. Balaji Zacharia, Jojo Inassi, Dhiyaneswaran Subramaniyan. Sandesh Pacha Unipolar Austin Moore’s Prosthesis Versus Cemented Bipolar Arthroplasty in Displaced Neck of Femur Fracture, in Elderly Patients Journal of Clinical and Diagnostic Research. 2018;12(8):RC01-RC04. 